\title{
DIVERGENCE AND FLUTTER INSTABILITY OF A STEPPED COLUMN LOADED BY THE FOLLOWER FORCE
}

\author{
Krzysztof Sokót \\ Institute of Mechanics and Machine Design Foundation \\ Czestochowa University of Technology \\ Czestochowa, Poland \\ sokol@imipkm.pcz.pl
}

\begin{abstract}
In this chapter the divergence and flutter instability problem of a stepped column is presented. The change of instability is obtained by application of the follower force on the free end of the discussed structure. The column is composed of three sections with different cross-sectional areas. The connection of odd and even sections is realized by means of pins strengthened with rotational springs. The proposed type of connection allows one to simulate the different methods of assembly or even the defect in the form of cracks. The instability problem is formulated on the basis of the Hamilton's principle and solved numerically. The results concern maximum divergence and flutter loads and the shape of characteristic curves.
\end{abstract}

Keywords: column, instability, loading capacity, vibrations

\section{Introduction}

In this manuscript, the instability phenomenon of a cantilever column loaded by the follower force is presented. The system is a slender one due to the high slenderness ratio. The proposed type of external load leads to divergence or flutter instability which depends on the follower factor. The nomenclature of the follower factor $\eta$ was suggested by Kordas and Życzkowski [1]. They have created four classes of the factor: $\eta<0$ - anti-follower force, $0<\eta<1$ - sub-follower force, $\eta=1$ - follower force, $\eta>1$ - super-follower force.

Theoretical as well as numerical studies were performed by many scientists from different scientific departments. In recent years, Przybylski and Sokół [2] have investigated the multimember column integrated with a piezoceramic element. The piezo rod was used for generation of compressive or tensional internal forces. The use of this element leads to the pre-stressing of the column. Authors have shown that the investigated supporting structure loses stability via divergence or flutter and that the prestressing causes the shift in divergence/flutter instability regions. The connection of the piezorod to the host structure was realized by means of the rotational spring with a linear characteristic. The spring model has also been applied to study the dynamics of bolted joints [3] and dynamic and stability of the robotic arms [4]. 
The use of rotational springs in the connection between chosen elements of the structure also allows one to simulate the defects in the connection such as cracks. Ostachowicz and Krawczuk [5] have proposed the exact solution for a single-sided and double-sided cracked beam on the basis of which the spring stiffness was obtained. Sokó [6] has studied the multi-member cracked system loaded by the external force with a constant line of action where the crack was modeled with a rotational spring. As a result, the characteristic curves and amplitude - vibration frequency relationship have been obtained.

The stepped columns have been studied with the use of different methods. Kukla [7] proposed the analytical simulation done with the Green's function while Zamorska et. al. [8] compared the solution with the Green's function to the ones from CATIA software. Studies on stepped columns have also been performed by Sokół [9].

Tomski and Uzny [10] have studied a column with a constant cross-sectional area strengthened by the spring at the fixed end and loaded by the generalized Beck's load. As a result of their work, the instability regions have been found. Sugiyama [11] has performed the experimental studies on flutter instability. The external load was induced by the real solid rocket motor located on the tip of the column. Finally, the flutter instability phenomenon was confirmed. The instability problem concerns not only slender systems but also different engineering structures such as sandwich panels $[12,13]$ which cover the supporting column and protects it from the environmental factors. The studies with consideration of different theories (Timoshenko, Euler) in relation to the columns with different slenderness factor were presented in [14, 15].

As a combination of the studies done by other researchers (stepped columns, connection stiffness of segments done with rotational spring, follower load) in this paper, the stepped cantilever column loaded by the external follower force is investigated. The considered structure is composed of three sections connected by the pins strengthened with rotational springs. The proposed method of connection allows one to simulate the different methods of assembly of even and odd sections and has not been previously discussed in stepped columns with divergence/flutter instability. The presented results concern the shape of characteristic curves (external load - vibration frequency) and divergence/flutter instability regions in relation to the different stiffness of rotational springs and cross-sectional area of sections. The obtained data can be used in construction of the nozzle supporting structure from which the fluid flows with high velocity.

\section{Boundary problem formulation}

The investigated slender stepped system is presented in Figure 1. As shown, the structure is loaded at the free end by the external force $P$. The follower factor $\eta$ is responsible for the change of line of action of force $P$. Sections have lengths $l_{1}, l_{2}, l_{3}$ respectively. Even and odd sections are connected by rotational springs. 
The springs have stiffness $C_{H}$ and $C_{L}$. The general boundary problem formulation allows one to simulate not only an influence of the rotational springs stiffness on instability and natural vibration frequency but also other parameters such as cross-sectional areas of sections or length of the elements.

The boundary problem has been formulated on the basis of the Hamilton's principle:

$$
\delta \int_{t_{1}}^{t_{2}}(T-V) d t+\int_{t_{1}}^{t_{2}} \delta L_{n} d t=0
$$

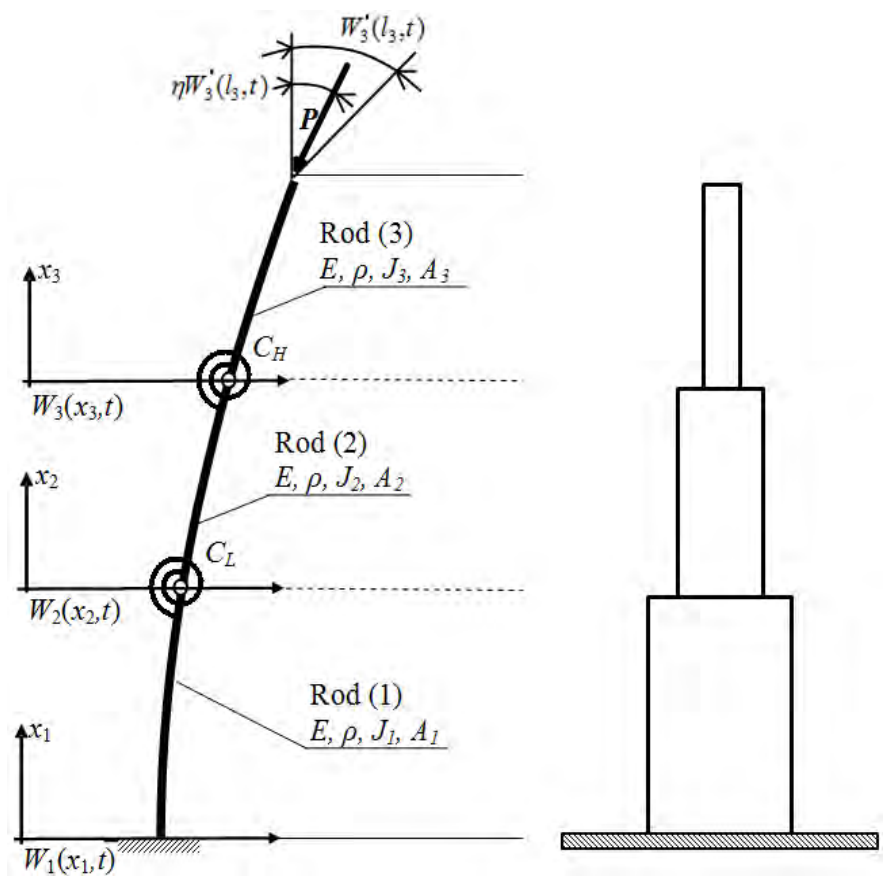

Fig. 1. Investigated system

The kinetic and potential energies and work are as follows:

$$
\begin{gathered}
V=\frac{1}{2}\left\{\sum_{i=1}^{3} \int_{0}^{l_{i}} E J_{i}\left[\frac{\partial^{2} W_{i}\left(x_{i}, t\right)}{\partial x_{i}^{2}}\right]^{2} d x\right\}-P \frac{1}{2}\left\{\sum_{i=1}^{3} \int_{0}^{l_{i}}\left[\frac{\partial W_{i}\left(x_{i}, t\right)}{\partial x_{i}}\right]^{2} d x\right\}+ \\
+\frac{1}{2} C_{H}\left(\left.\frac{\partial W_{3}\left(x_{3}, t\right)}{\partial x_{3}}\right|_{x_{3}=0}-\left.\frac{\partial W_{2}\left(x_{2}, t\right)}{\partial x_{2}}\right|^{x_{2}=l_{2}}\right)^{2}+ \\
+\frac{1}{2} C_{L}\left(\left.\frac{\partial W_{2}\left(x_{2}, t\right)}{\partial x_{2}}\right|_{x_{2}=0}-\left.\frac{\partial W_{1}\left(x_{1}, t\right)}{\partial x_{1}}\right|^{x_{1}=l_{1}}\right)^{2}
\end{gathered}
$$




$$
\begin{aligned}
& T=\frac{1}{2} \sum_{i=1}^{3} \int_{0}^{l_{i}} \rho A_{i}\left(\frac{\partial W_{i}\left(x_{i}, t\right)}{\partial t}\right)^{2} d x \\
& L_{n}=\left.\eta P \frac{\partial W_{3}\left(x_{3}, t\right)}{\partial x_{3}}\right|_{x_{3}=l_{3}} W_{3}\left(l_{3}, t\right)
\end{aligned}
$$

where: $E$ - Young's modulus, $J_{i}$ - moment of inertia, $A_{i}$ - cross-sectional area, $\rho$ - material density, $C_{H}, C_{L}$ - rotational spring stiffness, $P$ - external load, $W_{i}$ - transversal displacement, $\eta$ - follower factor.

Equations (2a-c) are being introduced into the Hamilton's principle - eq. (1). An integration and variation operation performed on eq. (1) lead inter alia to equations of motion in transversal direction which can be written in the form:

$$
E J_{i} \frac{\partial^{4} W_{i}\left(x_{i}, t\right)}{\partial x_{i}^{4}}+P \frac{\partial^{2} W_{i}\left(x_{i}, t\right)}{\partial x_{i}^{2}}+\rho A_{i} \frac{\partial^{2} W_{i}\left(x_{i}, t\right)}{\partial t^{2}}=0
$$

The geometrical boundary conditions (obtained on the basis of the geometry of the system) and natural ones (found from the Hamilton's principle after taking into account the geometrical boundary conditions) are given below:

$$
\begin{gathered}
\left.W_{1}\left(x_{1}, t\right)\right|_{x_{1}=0}=0 \\
\left.\frac{\partial W_{1}\left(x_{1}, t\right)}{\partial x_{1}}\right|_{x_{1}=0}=0 \\
\left.E J_{3} \frac{\partial^{2} W_{3}\left(x_{3}, t\right)}{\partial x_{3}^{2}}\right|^{x_{3}=l_{3}}=0 \\
\left.\frac{\partial W_{2}\left(x_{2}, t\right)}{\partial x_{2}}\right|_{x_{2}=0}=\left.\frac{\partial W_{1}\left(x_{1}, t\right)}{\partial x_{1}}\right|^{x_{1}=l_{1}} \\
\left.\frac{\partial W_{3}\left(x_{3}, t\right)}{\partial x_{3}}\right|_{x_{3}=0}=\left.\left.\frac{\partial W_{2}\left(x_{2}, t\right)}{\partial x_{2}}\right|^{x_{1}=l_{2}}\right|_{x_{2}=0}-\left.S_{2} \frac{\partial W_{2}\left(x_{2}, t\right)}{\partial x_{2}}\right|_{x_{2}=0}=0 \\
\left.E J_{1} \frac{\partial^{3} W_{1}\left(x_{1}, t\right)}{\partial x_{1}^{3}}\right|^{x_{1}=l_{1}}+\left.S_{1} \frac{\partial W_{1}\left(x_{1}, t\right)}{\partial x_{1}}\right|^{x_{1}=l_{1}}+ \\
-E J_{2} \frac{\partial^{3} W_{2}\left(x_{2}, t\right)}{\partial x_{2}^{3}} \mid+
\end{gathered}
$$




$$
\begin{aligned}
& \left.E J_{2} \frac{\partial^{3} W_{2}\left(x_{2}, t\right)}{\partial x_{2}^{3}}\right|^{x_{2}=l_{2}}+\left.S_{2} \frac{\partial W_{2}\left(x_{2}, t\right)}{\partial x_{2}}\right|^{x_{2}=l_{2}}+ \\
& -\left.E J_{3} \frac{\partial^{3} W_{3}\left(x_{3}, t\right)}{\partial x_{3}^{3}}\right|_{x_{3}=0}-\left.S_{3} \frac{\partial W_{3}\left(x_{3}, t\right)}{\partial x_{3}}\right|_{x_{3}=0}=0 \\
& -\left.E J_{2} \frac{\partial^{2} W_{2}\left(x_{2}, t\right)}{\partial x_{2}^{2}}\right|_{x_{2}=0}+C_{L}\left(\left.\frac{\partial W_{2}\left(x_{2}, t\right)}{\partial x_{2}}\right|_{x_{2}=0}-\left.\frac{\partial W_{1}\left(x_{1}, t\right)}{\partial x_{1}}\right|^{x_{1}=l_{1}}\right)=0 \\
& \left.E_{1} J_{1} \frac{\partial^{2} W_{1}\left(x_{1}, t\right)}{\partial x_{1}^{2}}\right|^{x_{1}=l_{1}}-C_{L}\left(\left.\frac{\partial W_{2}\left(x_{2}, t\right)}{\partial x_{2}}\right|_{x_{2}=0}-\left.\frac{\partial W_{1}\left(x_{1}, t\right)}{\partial x_{1}}\right|^{x_{1}=l_{1}}\right)=0 \\
& -\left.E J_{3} \frac{\partial^{2} W_{3}\left(x_{3}, t\right)}{\partial x_{3}^{2}}\right|_{x_{3}=0}+C_{H}\left(\left.\frac{\partial W_{3}\left(x_{3}, t\right)}{\partial x_{3}}\right|_{x_{3}=0}-\left.\frac{\partial W_{2}\left(x_{2}, t\right)}{\partial x_{2}}\right|^{x_{2}=l_{2}}\right)=0 \\
& \left.E J_{2} \frac{\partial^{2} W_{2}\left(x_{2}, t\right)}{\partial x_{2}^{2}}\right|^{x_{2}=l_{2}}-C_{H}\left(\left.\frac{\partial W_{3}\left(x_{3}, t\right)}{\partial x_{3}}\right|_{x_{3}=0}-\left.\frac{\partial W_{2}\left(x_{2}, t\right)}{\partial x_{2}}\right|^{x_{2}=l_{2}}\right)=0 \\
& \left.E J_{3} \frac{\partial^{3} W_{3}\left(x_{3}, t\right)}{\partial x_{3}^{3}}\right|^{x_{3}=l_{3}}+\left.P(1-\eta) \frac{\partial W_{3}\left(x_{3}, t\right)}{\partial x_{3}}\right|^{x_{3}=l_{3}}=0
\end{aligned}
$$

The formulated boundary problem is solved numerically by introduction of equations of motion into boundary conditions. This leads to the set of equations on the basis of which the matrix determinant can be found. Equating the matrix determinant to zero, one finds the transcendental equation for external load - natural vibration frequency relationship. The presented mathematical model can correspond to the real structure at the end of which the nozzle is placed from where the fluid flows with high velocity.

\section{Results of numerical simulations}

For a more general problem description, the results of numerical simulations are presented in the non-dimensional form on the basis of the following relations: 


$$
\begin{aligned}
& p=\frac{P l^{2}}{E J_{1}}, c_{H}=\frac{C_{H} l}{E J_{1}}, c_{L}=\frac{C_{L} l}{E J_{1}}, d_{i}=\frac{l_{i}}{l}, \\
& \mu_{21}=\frac{E J_{2}}{E J_{1}}, \mu_{32}=\frac{E J_{3}}{E J_{2}}, \omega=\sqrt{\Omega^{2} \frac{\rho A_{1} l^{4}}{E J_{1}}}
\end{aligned}
$$

It is assumed that the total length of the column is $l=l_{1}+l_{2}+l_{3}$ and that the cross-sectional area of the segment 1 is invariable. The rods are made of the same material which is why the Young's modulus and material density are not indexed.

At first, the verification of the proposed mathematical model was done on the basis of which the characteristic curves and maximum external force magnitude have been calculated - see Figure 2. The column was composed of identical sections connected by springs of infinite stiffness. At $\eta=0$, the maximum external load magnitude is $p=2.465$. An increase of the follower factor results in an increase of the maximum loading capacity and finally at $\eta>0.5$ causes the change of the instability type from divergence to flutter. At $\eta=1$, the maximum applied load is $p=20.05$ - Beck's load.

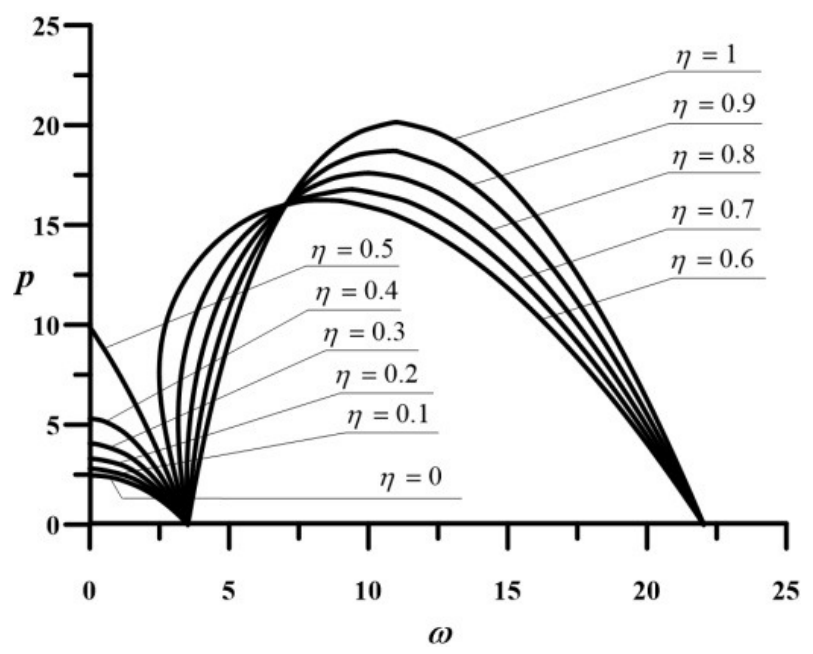

Fig. 2. Characteristic curves of the reference structure - constant cross-sectional area system $\left(c_{H}=c_{L}=10^{3}, d_{1}=d_{2}=d_{3}=0.33, \mu_{21}=\mu_{32}=1\right)$

An influence of the change of a cross-sectional area of segments 2 and 3 on instability type and characteristic curves shape was plotted in Figure 3. At all investigated $\eta$ magnitudes an increase of the free vibration frequency has been observed in relation to Figure 2. Additionally, the reduction of both divergence and flutter maximum external load magnitude is present regardless to $\eta$. 


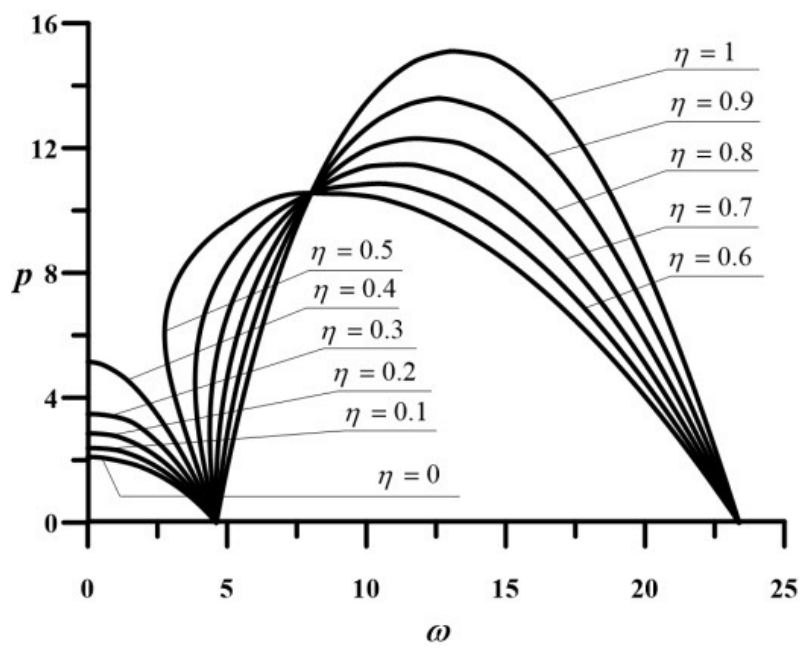

Fig. 3. Characteristic curves of the reference structure - stepped system $\left(c_{H}=c_{L}=10^{3}, d_{1}=d_{2}=d_{3}=0.33, \mu_{21}=0.75, \mu_{32}=0.5\right)$

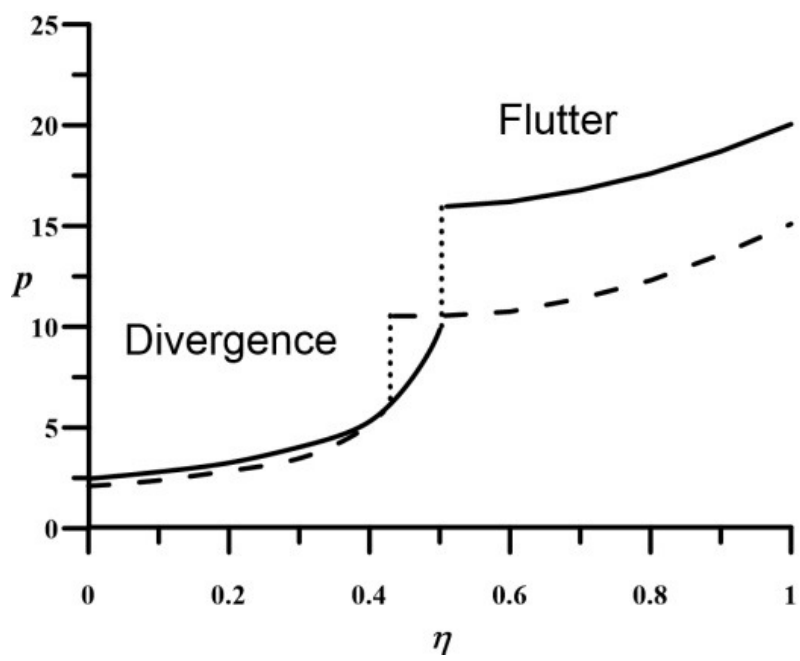

Fig. 4. Divergence and flutter instability regions of stepped column $\left(\mu_{21}=0.75, \mu_{32}=0.5\right)$ and continuous one $\left(\mu_{21}=\mu_{32}=1\right)$

The common point through which characteristic curves are passing is shifted to higher vibration frequency magnitudes and is present at lower $p$ in relation to configuration presented in Figure 1. The difference in the instability regions has been plotted in Figure 4. The continuous line corresponds to the system with even sections while the dashed one stands for the stepped structure. 


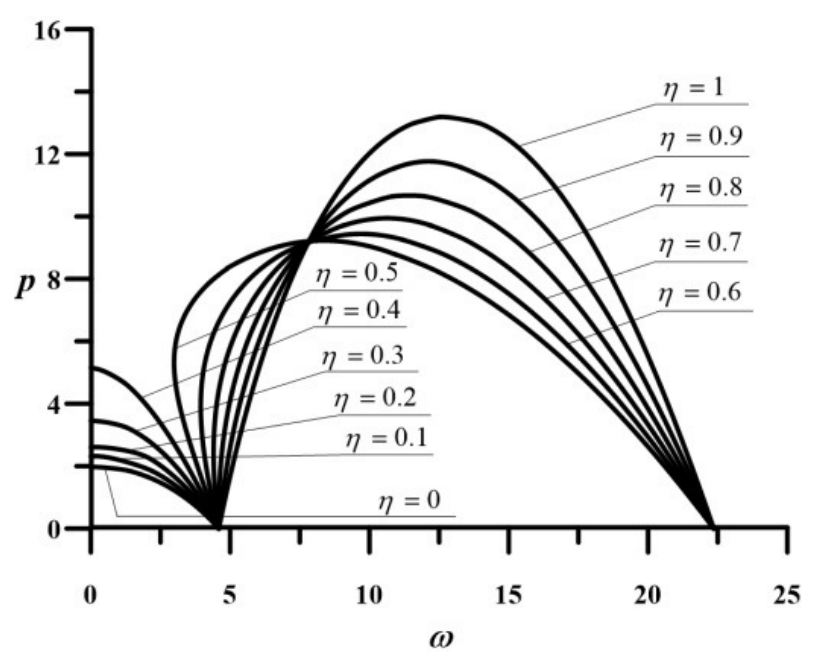

Fig. 5. Characteristic curves of the stepped column $\left(c_{H}=10, c_{L}=10^{3}, d_{1}=d_{2}=d_{3}=0.33, \mu_{21}=0.75, \mu_{32}=0.5\right)$

It has been shown that the reduction of a cross-sectional area of segments 2 and 3 affects not only vibration frequency and loading capacity but also reduces the divergence instability area. Moreover, the difference between the maximum divergence force and the lowest flutter one is smaller when the stepped system is used. It can be concluded that the proper selection of cross-sectional areas of segments leads to the design of the structure which meets the specific requirements of the place of installation and allows one to control the type of instability.

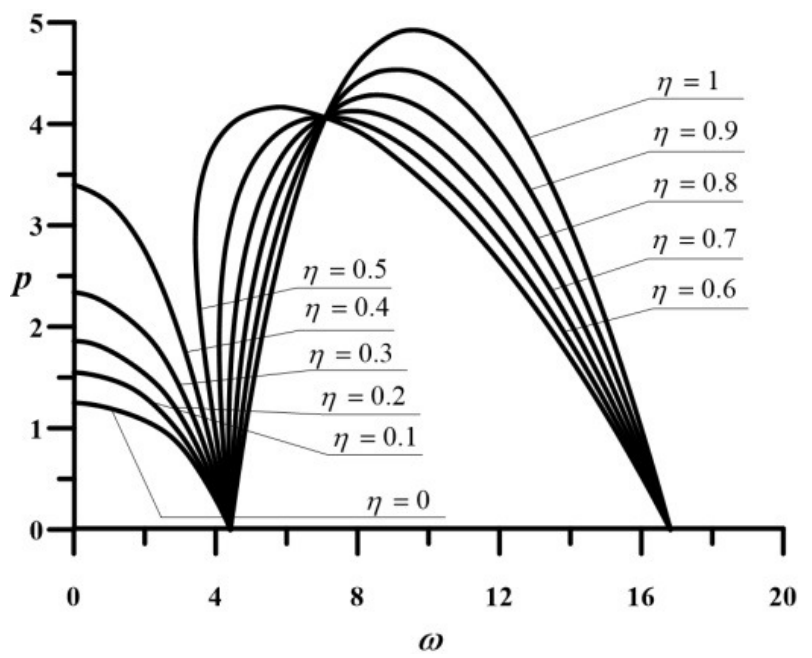

Fig. 6. Characteristic curves of the stepped column $\left(c_{H}=1, c_{L}=10^{3}, d_{1}=d_{2}=d_{3}=0.33, \mu_{21}=0.75, \mu_{32}=0.5\right)$ 
The reduction of the stiffness of connection between sections 2 and 3 has been plotted in Figures 5 and 6 . The reduction of spring $c_{H}$ causes the reduction of the vibration frequency magnitude as well as maximum divergence and flutter loading capacity in relation to the reference stepped column configuration. The common point is being shifted to the higher vibration frequency magnitudes and to the lower external load $p$. The difference between the maximum divergence force and the minimum flutter one is getting smaller at weaker connection stiffness of sections 2 and 3 .

When the reduction of the stiffness of the connection of segments 1 and 2 is taken into account, Figures 7 and 8 should be discussed. The reduction of the $c_{L}$ parameter down to 10 changes the maximum divergence as well as flutter loading. The divergence maximum loading capacity is now smaller than in the $c_{H}=10$ case. When the flutter instability is considered, it has been noticed that the second free vibration frequency as well as the maximum external load magnitude are greater at $c_{H}=10$. The location of the common point at $c_{L}=10$ is now shifted to the greater $p$ magnitude and has a slightly greater vibration frequency. In the case of reduction of $c_{L}$ rotational spring stiffness down to 1 , the lowest maximum divergence loads were obtained as well as first free vibration frequencies. Now, the divergence instability is present even at $\eta=0.6$.

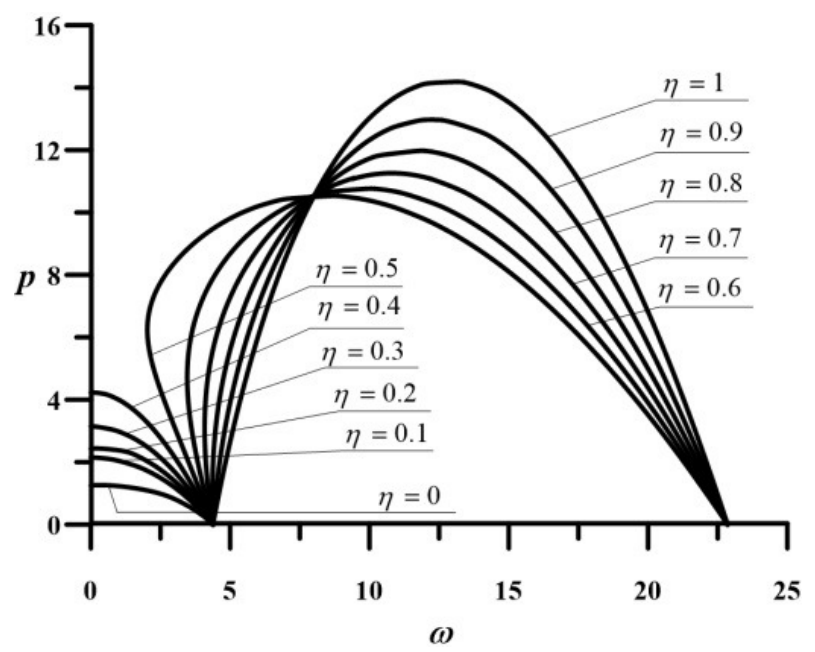

Fig. 7. Characteristic curves of the stepped column $\left(c_{L}=10, c_{H}=10^{3}, d_{1}=d_{2}=d_{3}=0.33, \mu_{21}=0.75, \mu_{32}=0.5\right)$

The second free vibration frequency magnitude is greater than it is in the presented case at $c_{H}=1$. The flutter instability at $c_{L}=1$ leads to greater external load magnitudes than at $c_{H}=1$. The difference between maximum divergence load and the minimum flutter one is becoming greater at smaller $c_{L}$ stiffness. 


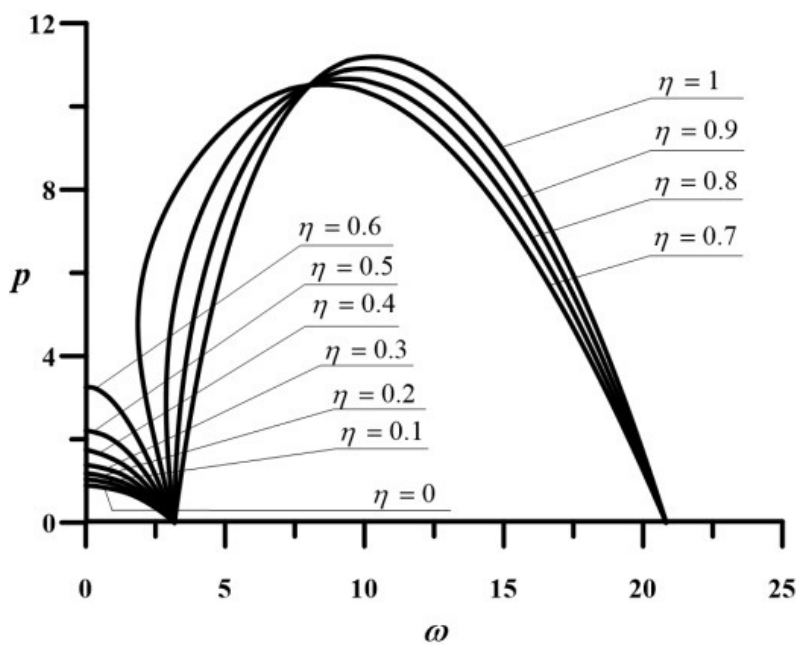

Fig. 8. Characteristic curves of the stepped column $\left(c_{L}=1, c_{H}=10^{3}, d_{1}=d_{2}=d_{3}=0.33, \mu_{21}=0.75, \mu_{32}=0.5\right)$

An influence of the rotational springs stiffness on loading capacity of the discussed system at different $\eta$ is presented in Figure 9. The $\eta$ parameter magnitude has been chosen in the way that the instability type is constant regardless of spring stiffness. The continuous lines correspond to the divergence instability while the dashed ones are for flutter. At divergence instability, the reduction of rotational spring stiffness at the beginning causes a very slow reduction of maximum external load magnitude.

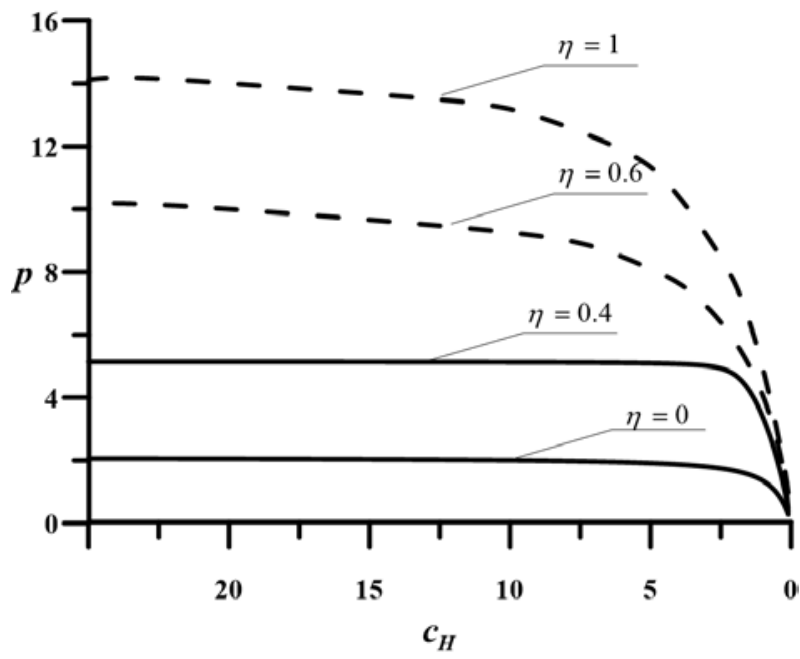

Fig. 9. Influence of $c_{H}$ stiffness on loading capacity $\left(c_{L}=10^{3}, d_{1}=d_{2}=d_{3}=0.33, \mu_{21}=0.75, \mu_{32}=0.5\right)$ 


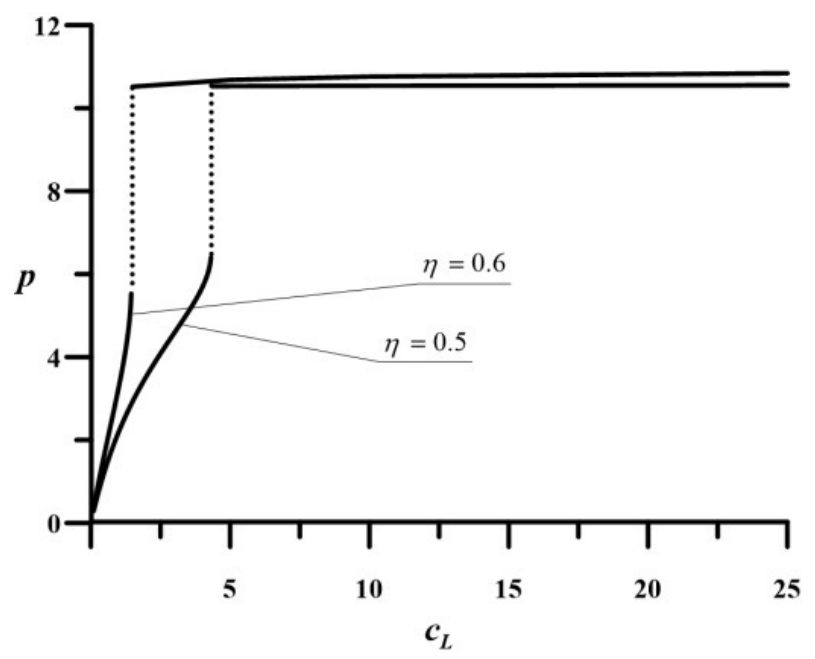

Fig. 10. Influence of $c_{L}$ stiffness on instability $\left(c_{H}=10^{3}, d_{1}=d_{2}=d_{3}=0.33, \mu_{21}=0.75, \mu_{32}=0.5\right)$

Further reduction of spring stiffness down to magnitudes smaller than 2.5 results in a rapid decrease of loading capacity. At flutter instability, the maximum external load magnitude decreases sooner but the reduction does not occur as rapidly as the divergence instability does. In this case, the external load magnitude which corresponds to the one of the reference systems will be obtained at a stiffer spring than at smaller $\eta$. When lower rotational spring $c_{L}$ is discussed, the analogous situation to the described above takes place.

In Figure 10 an influence of the rotational spring stiffness on the type of instability has been plotted. An increasing stiffness of the spring, stiffens the connection of sections which results in a change of initial divergence instability to the flutter one. At the greater $\eta$ the change of instability will occur at a smaller spring stiffness. The similar tendency can be found at other parameters of the system.

\section{Conclusions}

In this paper, the divergence and flutter instability problem of a stepped column was presented. The change of instability was obtained by application of the follower force on the free end of the structure. The column is composed of three sections with different cross-sectional areas. The detailed conclusions were made in the discussion of the results of the numerical simulations section. As a summary it can be stated that:

- an introduction of rotational springs allows one to simulate the different connection stiffness of even and odd sections,

- the weaker connection stiffness the smaller loading capacity of both flutter and divergence in relation to the proposed reference system, 
- the control of rotational spring stiffness allows one to control the free vibration frequencies and loading capacity,

- by means of proper selection of connection stiffness, the control of divergence/ /flutter instability regions can be obtained.

In future studies, an influence of length of sections should be discussed. Experimental verification of the studies can also be done.

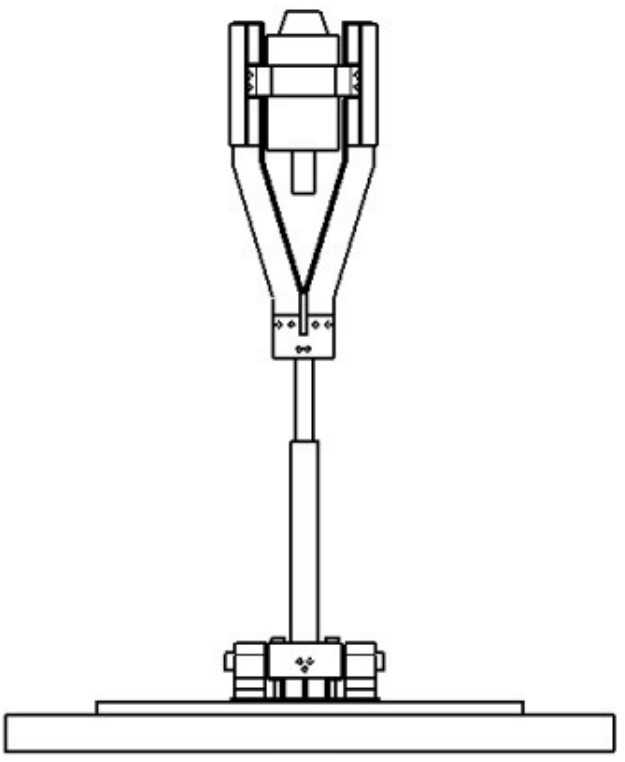

Fig. 11. Proposed construction of the experimental stand

The exemplary experimental stand is presented in Figure 11. It contains inter alia: the base with the mounting bracket, test column and a scale RC-model jet engine which will simulate the follower load.

\section{References}

[1] Kordas Z., Życzkowski M., On the loss of stability of a rod under-super tangential force, Archiwum Mechaniki Stosowanej 1963, 21(1), 7-31.

[2] Przybylski J., Sokół K., The control of divergence and flutter instability of the geometrically non-linear system by means of the piezoelectric force, [in:] Free Vibrations and Stability of Slender Systems Subjected to Conservative or Non-conservative Load, WNT, 2012 (in Polish).

[3] Wang J.H., Chuang S.C., Reducing errors in the identification of structural joint parameters using error functions, Journal of Sound and Vibration 2004, 273(1-2), 295-316.

[4] Liu Y., Li W., Yang X., Wang Y., Dynamic and stability of harmonic driving flexible cartesian robotic arm with bolted joints based on the sensitivity and multiple scales method, Mathematical Problems in Engineering, http://dx.doi.org/10.1155/2015/795101.

[5] Ostachowicz W.M., Krawczuk M., Analysis of the effect of cracks on the natural frequencies of a cantilever beam, Journal of Sound and Vibration 1991, 150, 191-201. 
[6] Sokół K., Linear and nonlinear vibrations of a column with an internal crack, Journal of Engineering Mechanics 2014, 140(5), http://dx.doi.org/10.1061/(ASCE)EM.1943-7889.0000719.

[7] Kukla S., Free vibrations and stability of stepped columns with cracks, Journal of Sound and Vibration 2009, 319(3-5), 1301-1311.

[8] Sokół K., Instabilitty of a stepped column loaded by the external follower force, Engineering Mechanics 2017, 894-897.

[9] Zamorska I., Cekus D., Miara M., Effect of crack parameters on free vibrations of the Berrnoulli-Euler beam, Journal of Applied Mathematics and Computational Mechanics 2015, 14(4), 167-174.

[10] Tomski L., Uzny S., The regions of flutter and divergence instability of a column subjected to Beck's generalized load taking into account the torsional flexibility of the loaded end of the column, Research Mechanics Communications 2011, 38, 95-100.

[11] Sugiyama Y., Katayama K., Kinoi S., Flutter of cantilevered column under rocket thrust, Journal of Aerospace Engineering 1995, 8(1), 9-15.

[12] Pozorska J., Pozorski Z., Analysis of the failure mechanism of the sandwich panel at the supports, XXI Polish-Slovak Scientific Conference Machine Modeling and Simulations MMS 2016, DOI: 10.1016/j.proeng.2017.02.213.

[13] Pozorska J., Pozorski Z., New insights into classical solutions of the local instability of the sandwich panels, Problem Proceedings of The International Conference On Numerical Analysis And Applied Mathematics 2015, DOI: 10.1063/1.4952323.

[14] Sokół K., Uzny S., The Bernoulli-Euler and Timoshenko theories in the context of research on the characteristic curves of column with different boundary conditions, AIP Conference Proceedings, 2014, (1648), DOI: 10.1063/1.4913091.

[15] Uzny S., Sokół K., Osadnik M., Influence of amplitude on free vibration frequency of a partially tensioned column, Engineering Mechanics 2016, 562-565. 